\title{
Amplified fragment length polymorphism (AFLP) analysis of Clostridium novyi, C. perfringens and Bacillus cereus isolated from injecting drug users during 2000
}

\author{
J. MCLAUCHLIN, J. E. SALMON†, S. AHMED§, J. S. BRAZIER $\pitchfork$, M. M. BRETT, R. C. GEORGE* \\ and J. HOOD\|
}

Food Safety Microbiology Laboratory, Division of Gastrointestinal Infections and * Respiratory and Systemic Infection Laboratory, PHLS Central Public Health Laboratory, 61 Colindale Avenue, London NW9 5HT, $\dagger$ Department of Medical Microbiology and Public Health Laboratory and $\$ P H L S$ Anaerobe Reference Unit, University Hospital of Wales, Heath Park, Cardiff CF14 4XW, §Greater Glasgow Health Board, 350 Vincent Street, Glasgow G3 8YU and ||Department of Clinical Microbiology, Glasgow Royal Infirmary, 84 Castle Street, Glasgow G4 OSF

\begin{abstract}
As part of the follow-up investigations associated with an outbreak of severe illness and death among illegal injecting drug users during 2000, 43 cultures of Clostridium novyi type A, $40 C$. perfringens type A and 6 isolates of Bacillus cereus were characterised by amplified fragment length polymorphism (AFLP) analysis. Among the 43 C. novyi isolates, 23 different AFLP profiles were detected. The same AFLP profile was detected in isolates from 18 drug users investigated during 2000 from Scotland, England, the Republic of Ireland and Norway and a wound from a patient in 2000 who was not identified as a drug user. Unique AFLP profiles were obtained from four drug users from England and the Republic of Ireland, 10 historical isolates from culture collections, an isolate from food (1989) and three isolates from wounds $(1995,1991,1988)$. The $40 C$. perfringens isolates were from 13 drug users, the contents of one syringe and two samples of heroin. Sixteen AFLP types of $C$. perfringens were distinguished and there was little evidence for commonality among the isolates. The AFLP types of $C$. perfringens from heroin differed and were unique. Six isolates of $B$. cereus were from four drug users and two samples of heroin. Four different AFLP patterns were distinguished. Three AFLP types were isolated from four drug users. B. cereus isolates from an aspirate and a heroin sample collected from the same drug user were identical, and were also indistinguishable from an isolate from a groin infection in a second drug user. The AFLP type of the isolate from a second and unrelated heroin sample was unique. The AFLP results showed no or very limited evidence for commonality between the different isolates of $B$. cereus and $C$. perfringens. In marked contrast, the $C$. novyi isolates from the majority of the drug users during 2000 were homogeneous, suggesting a common source or clonal selection of a $C$. novyi type, or both, which either had an adaptive advantage in spore germination, survival or growth following the drug preparation and the injection procedure, or produced a more severe clinical presentation.
\end{abstract}

\section{Introduction}

A range of viral and bacterial infections is associated with illegal injecting drug use [1-3]. Endospore-

Received 15 May 2002; accepted 20 July 2002.

Corresponding author: Dr J. McLauchlin (e-mail: jmclauchlin@phls.org.uk). forming bacteria, including Clostridium botulinum [4-6], C. tetani [7] and Bacillus anthracis [8] are increasingly recognised as a group of agents that infect injecting drug users. These agents are particularly problematic in this patient group because of their occurrence in the intestinal tract, widespread distribution in the environment, the robust nature of bacterial endosopores and their resistance to the process of drug preparation [9]. 
In 2000, an unusual increase of morbidity and mortality was reported among illegal injecting drug users in the UK and Ireland. During the intensive investigation more than 108 cases (at least 43 deaths) were recognised between 1 April and 31 August 2000 [10-12]. C. novyi type A was the likely cause of the more serious illness [12-15], although other endospore-forming bacteria were also associated with some cases including $C$. perfringens and B. cereus [15-19]. In addition, six suspected cases of wound botulism were also reported in injecting drug users during 2000 and these represent the first recognition of this condition in the UK $[16,19,20$, PHLS unpublished data].

Amplified fragment length polymorphism (AFLP) has been applied successfully to bacteria from a wide variety of different genera [21-25]. The method involves restriction endonuclease digestion of total purified chromosomal DNA followed by ligation of the resulting fragments to a double-stranded oligonucleotide adapter complementary to the base sequence of the restriction site. The adapters are designed such that the original restriction site is not restored after ligation, thus preventing further restriction digestion. Selective amplification by PCR of sets of these fragments is achieved with primers corresponding to the contiguous base sequences in the adapter, the restriction site plus one or more nucleotides in the original target DNA. The resulting PCR-amplified DNA fragments are then analysed by gel electrophoresis.

It was reported previously that AFLP could be applied successfully for epidemiological typing of $B$. cereus and $C$. perfringens cultures from cases of food poisoning $[26,27]$. The purpose of the present study was to use AFLP for molecular epidemiological investigations of isolates of $B$. cereus and C. perfringens from injecting drug users during the 2000 outbreak, and to apply this technique to $C$. novyi isolates.

\section{Materials and methods}

\section{Sources of cultures and case definitions}

Isolates of $C$. novyi, $C$. perfringens and B. cereus were obtained as pure cultures or other material referred directly to the PHLS laboratories, from clinical microbiology laboratories, or were from culture collections; origins of isolates are shown in Tables 1-4 [28].

Clinical isolates of $C$. novyi, $C$. perfringens and $B$. cereus were from illegal injecting drug users in England, Scotland, the Republic of Ireland and Norway: each patient was designated with a number. All cases were in individuals who injected heroin extravascularly (i.e., subcutaneously or intramuscularly) and who presented to hospital with moderate to severe inflammation in soft tissue at an injection site unless otherwise stated. The cases were categorised as described elsewhere [15] as follows: definite, a case with an abscess or other significant inflammation at an injecting site, and both a severe inflammatory process (large abscess/extensive oedema) at or around the injecting site, and a severe systemic inflammatory reaction with evidence of multi-organ failure and a high white cell count; probable, a case with an abscess or other significant inflammation at an injecting site, and either a severe inflammatory process (large

Table 1. Origins and characterisation of C. novyi isolates from drug users

\begin{tabular}{|c|c|c|c|c|c|c|}
\hline Patient no. & Sex & Country & Case status & Onset/ isolation date & Source of isolates & AFLP type \\
\hline 5 & M & England & Possible & December 1999 & Abscess aspirate & B \\
\hline 6 & M & England & Definite & February 2000 & Tissue & A \\
\hline 7 & $\mathrm{~F}$ & England & Probable & April 2000 & Tissue & A \\
\hline \multirow[t]{2}{*}{$8^{*}$} & $\mathrm{~F}$ & Scotland & Definite & April 2000 & PM tissue & A \\
\hline & & & & & PM tissue & A \\
\hline 9 & M & England & Definite & May 2000 & PM injection site & A \\
\hline 10 & M & Scotland & Definite & May 2000 & PM tissue & A \\
\hline 11 & M & Scotland & Definite & May 2000 & PM kidney & A \\
\hline 12 & M & Scotland & Definite & May 2000 & AM tissue & A \\
\hline 13 & $\mathrm{~F}$ & Scotland & Probable & May 2000 & AM tissue & A \\
\hline 14 & $\mathrm{~F}$ & Scotland & Probable & May 2000 & AM tissue & A \\
\hline $3^{\dagger}$ & M & Scotland & Definite & May 2000 & AM tissue & A \\
\hline 24 & NK & Republic of Ireland & Definite & May 2000 & PM tissue & A \\
\hline $15^{*}$ & M & Scotland & Definite & June 2000 & AM tissue & A \\
\hline 16 & $\mathrm{~F}$ & Scotland & Probable & June 2000 & PM tissue & A \\
\hline $17^{*}$ & M & Scotland & Probable & June 2000 & PM tissue & $\mathrm{A}$ \\
\hline $18^{*}$ & M & Scotland & Definite & June 2000 & AM tissue & A \\
\hline 19 & M & Scotland & Probable & June 2000 & PM blood & A \\
\hline 20 & M & England & Possible & July 2000 & Calf skin & $\mathrm{C}$ \\
\hline 21 & $\mathrm{~F}$ & Scotland & Definite & August 2000 & PM tissue & A \\
\hline 22 & M & Norway ${ }^{*}$ & Definite & August 2000 & Tissue & A \\
\hline 23 & $\mathrm{~F}$ & Republic of Ireland & Probable & October 2000 & Calf abscess & $\mathrm{D}$ \\
\hline 34 & M & England & Probable & January 2001 & Tissue & $\mathrm{K}$ \\
\hline
\end{tabular}

NA, not applicable; NK, not known; M, male; F, female; AM, ante-mortem; PM, isolates collected at necropsy.

${ }^{*} C$. perfringens also isolated from these patients.

${ }^{\dagger} B$. cereus also isolated from this patient.

*As described by Maagaard et al. [28]. 
Table 2. Origins and characterisation of other C. novyi isolates

\begin{tabular}{|c|c|c|c|}
\hline Culture no. & $\begin{array}{l}\text { Year of } \\
\text { isolation }\end{array}$ & Source of isolate & $\begin{array}{c}\text { AFLP } \\
\text { type }\end{array}$ \\
\hline NCTC 6738 & 1944 & NK & $\mathrm{E}$ \\
\hline NCTC 538 & 1920 & Gas gangrene & $\mathrm{F}$ \\
\hline NCTC 13029 & NK & Type strain (ATCC 17861) & G \\
\hline CN 1376 & 1941 & War wound (Germany) & M \\
\hline CN 926 & $1944^{*}$ & Human mild infection of thigh (Australia) & $\mathrm{N}$ \\
\hline CN 902 & $1943^{*}$ & Human gas gangrene (Middle East) & $\mathrm{O}$ \\
\hline $\mathrm{CN} 930$ & $1944^{*}$ & 'Fatal case' (Australia) & $\mathrm{P}$ \\
\hline CN 1498 & $1946^{*}$ & Human post-operation gas gangrene (UK) & $\mathrm{J}$ \\
\hline CN 870 & 1918 & War wound (South Africa) & Q \\
\hline $\mathrm{CN} 925$ & $1944^{*}$ & Fatal human gas gangrene (New Guinea) & $\mathrm{R}$ \\
\hline R 4502 & 1991 & Wound, UK & $\mathrm{H}$ \\
\hline R 3140 & 1988 & Wound, UK & $\mathrm{I}$ \\
\hline R 8711 & 1995 & Blood, UK & $\mathrm{L}$ \\
\hline R14386 & 2000 & Human leg abrasion, $\mathrm{UK}^{\dagger}$ & A \\
\hline ED 15485 & NK & Horse, grass sickness & $\mathrm{S}$ \\
\hline ED 15486 & NK & Hare & $\mathrm{T}$ \\
\hline ED 15487 & NK & Horse, grass sickness & $\mathrm{U}$ \\
\hline ED 15489 & NK & Horse, grass sickness & $\mathrm{V}$ \\
\hline ED 15490 & NK & Horse, grass sickness & W \\
\hline R3738 & 1989 & Food, UK & $\mathrm{J}$ \\
\hline
\end{tabular}

NCTC, National Collection of Type Cultures, London; CN, Wellcome Bacterial Collection, held in the NCTC, London; R, PHLS Anaerobe Reference Unit, Cardiff PHL; ED, Department of Medical Microbiology, University of Edinburgh; NK, not known.

* Year of deposition.

${ }^{\dagger}$ As described by McGuigan and Roworth [41].

Table 3. Origins and characterisation of $C$. perfringens isolates

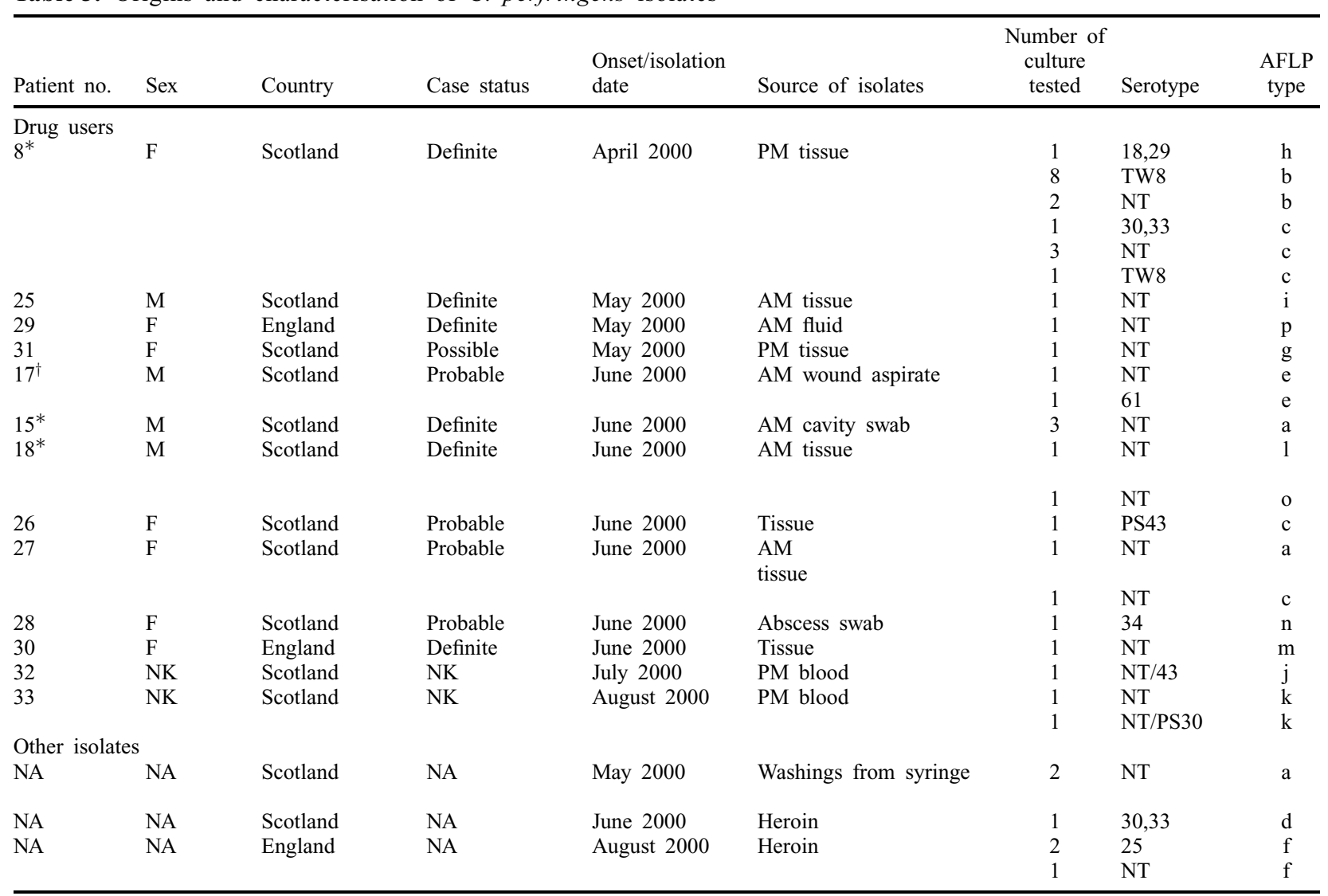

NA, not applicable; NT, non-typable; NK, not known.

${ }^{*} C$. novyi also isolated from these patients.

${ }^{\dagger} C$. novyi, $C$. scindens and C. perfringens isolated from this patient.

abscess/extensive oedema) at or around the injecting site, or a severe systemic inflammatory reaction with evidence of multi-organ failure and a high white cell count; or possible, a case with an abscess or other significant inflammation at an injecting site.
Additional isolates from heroin ( $B$. cereus and $C$. perfringens) and from used syringe residues $(C$. perfringens) from England or Scotland in 2000 were also included. Because of the lack of comparitors for characterising the $C$. novyi isolates, previously depos- 
Table 4. Origins and characterisation of B. cereus isolates

\begin{tabular}{|c|c|c|c|c|c|c|c|}
\hline Patient no. & Sex & Country & Case status & $\begin{array}{l}\text { Onset/isolation } \\
\text { date }\end{array}$ & $\begin{array}{l}\text { Source of } \\
\text { isolates }\end{array}$ & Serotype & $\begin{array}{l}\text { AFLP } \\
\text { type }\end{array}$ \\
\hline \multicolumn{8}{|l|}{ Drug users } \\
\hline 1 & M & England & $\mathrm{NA}^{*}$ & June 2000 & Blood & NT & I \\
\hline 2 & M & Scotland & Probable & June 2000 & Aspirate & NT & II \\
\hline $3^{*}$ & $\mathrm{M}$ & Scotland & Definite & May 2000 & Tissue & 11 & III \\
\hline 4 & $\mathrm{~F}$ & Scotland & Probable & June 2000 & Groin tissue & NT & $\mathrm{II}^{-1}$ \\
\hline \multicolumn{8}{|c|}{ Other isolates } \\
\hline NA & NA & England & NA & June 2000 & Heroin & NT & IV \\
\hline $\mathrm{NA}^{\dagger}$ & NA & Scotland & NA & June 2000 & Heroin & NT & II \\
\hline
\end{tabular}

NA, not applicable; NT, non-typable; NK, not known.

${ }^{*}$ C. novyi was also isolated from this patient.

${ }^{\dagger}$ Heroin collected from patient 2 [18].

No local inflammation around injection site.

ited cultures from other human or animal infections and one from food were analysed. These isolates were obtained from the culture collection of the PHLS Anaerobe Reference Unit; the National Collection of Type Cultures (NCTC), PHLS Central Public Health Laboratory, London; the Wellcome Bacterial Collection (also held at the NCTC); and from the Department of Medical Microbiology, University of Edinburgh (Table 2).

\section{C. novyi cultures}

The identity of all C. novyi type A isolates was confirmed according to conventional criteria [29] that included: spreading growth on blood agar, strong haemolysis on blood agar, lecithinase and lipase reactions positive on egg-yolk agar, and variable indole production. The identity of isolates was further confirmed by $\geqslant 98 \% 16 \mathrm{~S}$ rDNA sequence identity of a c. 1500-bp sequence of C. novyi type A strain NCTC 538 (EMBL database accession no. X68188) determined by methods described previously [30].

DNA was extracted from $C$. novyi cultures as described previously for $B$. cereus and $C$. perfringens [26, 27]. AFLP analysis was performed with either HindIII [24] or EcoR1 digestion [22]. For the HindIII digestion, ligation was as described previously [26, 27] and four separate PCR amplifications were performed with primers HI-A, HI-C, HI-G and HI-T (5'-GGTAT GCGACAGAGCTTA-3'， 5'-GGTATGCGACAGAGC TTC-3', 5'-GGTATGCGACAGAGCTTG-3' and 5'GGTATGCGACAGAGCTTT-3' respectively). Identical AFLP conditions were used for the EcoRI digestion, except the ligation adapters were 5'-CTCGTAGACT GCGTACC-3' and 5'-AATTGGTACGCAGTCTAC-3'. Four separate PCR amplifications were performed on the ligated and digested DNA with primers EC-A, EC-C, EC-G and EC-T (5'-GACTGCGTACCAATTCA-3', 5' GACTGCGTACCAATTCC-3'， 5'-GACTGCGTACCA ATTCG-3', and 5'-GACTGCGTACCAATTCT-3', respectively).
Brief epidemiological details on the origins of the isolates are shown in Tables 1 and 2 .

\section{C. perfringens cultures}

The identity of all $C$. perfringens type A cultures was confirmed by characteristic colonial morphology on Colombia blood agar and a positive Nagler reaction. All cultures were analysed by serotyping [31-34]. DNA was prepared as described previously and tested for amplification of fragments of the $\alpha$-toxin and enterotoxin genes [35] and by AFLP analysis [26]. The latter procedure comprised digestion of DNA with HindIII, ligation with adapters ADH1 and $\mathrm{ADH} 2$ followed by amplification with primer HI-G. Brief epidemiological information on the origins of the isolates is shown in Table 3.

\section{B. cereus cultures}

The identity of $B$. cereus cultures was confirmed by: characteristic colonial morphology on Columbia blood agar and Kendall's medium (lecithinase production and no fermentation of mannitol); the production of acid and gas from glucose but not xylose, mannitol or arabinose; resistance to $\gamma$ phage and penicillin; motility at $30^{\circ} \mathrm{C}$; and characteristic Gram and spore stains [36]. All cultures were analysed by serotyping of flagellar antigens [37,38] and characterised by AFLP analysis as described previously [27]. AFLP analysis comprised the digestion of total DNA with HindIII, ligation with adapters $\mathrm{ADH} 1$ and $\mathrm{ADH} 2$ followed by amplification with primer HI-A. Brief epidemiological details on the origins of the isolates are shown in Table 4.

\section{Analysis of DNA fragments}

DNA fragments produced by PCR were separated on $1 \%$ ( $\alpha$-toxin and enterotoxin PCR for $C$. perfringens), $1.5 \%$ (AFLP of $C$. novyi and C. perfringens) or $2 \%$ (AFLP of B. cereus) agarose-ethidium bromide electrophoresis gels, observed with UV transillumination and fluorescent bands were recorded with type 667 film 
(Polaroid, St Albans, UK). Images of the ethidium bromide-stained gels were analysed with Bionumerics version 2.0 (Applied Maths, Kortrijk, Belgium), by the unweighted pair group method using arithmetic means (UPGMA) with the Dice coefficient. Capital letters ( $C$. novyi), lower case letters (C. perfringens) or roman numerals (B. cereus) arbitrarily designated different AFLP patterns. Each AFLP banding pattern was defined as having more than two bands different from any other profiles for $C$. perfringens and $B$. cereus as described previously [26,27]. The analysis of the $C$. novyi banding patterns is described later.

\section{Results}

\section{C. novyi cultures}

Forty-three isolates of $C$. novyi were analysed; 23 cultures were from 22 injecting drug users with $C$. novyi infection presenting during 1999 (one case), 2000 (20 cases) and 2001 (one case) (Table 1). Because AFLP typing of $C$. novyi had not been attempted previously, other unrelated cultures were also included for comparison and these comprised three isolates from the NCTC; seven cultures from the Wellcome Bacterial Collection; two wound, one blood and one food isolate deposited in the PHLS Anaerobe Reference Unit in 1988, 1989, 1991 and 1995; five isolates from animals; and one further isolate from a leg abrasion of a patient during 2000 who was not known to be a drug user (Table 2).

AFLP analysis was performed on DNA from the 43 cultures and amplified by PCR with eight primers (primers HI-A, HI-C, HI-G and HI-T for the HindIII digestion, and EC-A, EC-C, EC-G and EC-T with the EcoRI digestion). Satisfactory banding patterns were obtained with primers HI-C, HI-G, EC-A, EC-C and EC-T. Banding patterns of the amplified DNA obtained with HI-A, HI-T and EC-G were poor and indistinct and are not considered further. The numbers of DNA fragments per pattern obtained with the remaining five primers were: primers HI-C, HI-G and EC-T three-tofive fragments; primer EC-A, three-to-eight fragments; primer EC-C, four-to-six fragments. The numbers of different banding patterns differing by more than one band and obtained with these five primers were: 10 patterns (HI-C), 10 (HI-G), 18 (EC-A), 12 (EC-C) and 6 patterns (EC-T). Representative banding patterns for each of the primers are shown in Fig. 1.

The reproducibility of the system was assessed initially by testing duplicate DNA extractions from three cultures with all five primers; identical banding patterns were obtained from each isolate. Two separate isolates were obtained from patient 8 (Table 1) and both gave identical banding patterns with all five primers. All cultures were tested with primer HI-G on two or more occasions and eight cultures were tested with primers $\mathrm{HI}-\mathrm{C}, \mathrm{EC}-\mathrm{A}, \mathrm{EC}-\mathrm{C}$ and $\mathrm{EC}-\mathrm{T}$ on two or more occasions; identical banding patterns were obtained for each culture in all instances.

An overall similarity was calculated based on the five sets of AFLP results and all isolates were assigned to one of 23 AFLP types based on $>95 \%$ UPGMA/ Dice similarity (Tables 1 and 2). A multi-dimensional scaling diagram showing a combined representation of the five sets of UPGMA/Dice similarity matrices is shown in Fig. 2. Of the 22 infected drug users from whom $C$. novyi was isolated, 18 cases occurred within the outbreak period (1 April-31 August 2000) previously defined [15]. Of these, eight definite and five probable cases occurred in Scotland; one definite, one probable and one possible case occurred in England; one definite case occurred in the Republic of Ireland; and a further definite case occurred in Norway during the outbreak period [28]. Of the remaining four injection-related infections, one possible and one definite case occurred in England in December 1999 and February 2000 respectively, a probable case occurred in the Republic of Ireland in October 2000, and a probable case in England in January 2001 (Table $1)$.

Among $C$. novyi isolates from the 22 drug users, cultures from 17 of the 18 patients (from Scotland, England, the Republic of Ireland and Norway) during the April-August 'outbreak period' yielded AFLP pattern A: 11 of these cases had a definite and six a probable status. The $C$. novyi from the remaining case during the outbreak period (patient number 20) was of possible status and yielded a unique AFLP profile designated as type C. C. novyi cultures from three of the four patients outside the outbreak period (patients 5, 23 and 34; December 1999, October 2000 and January 2001, respectively) who were of possible or probable case status gave unique AFLP patterns designated as $\mathrm{B}, \mathrm{D}$ and $\mathrm{K}$. The $C$. novyi from the remaining patient, who was of definite status, and whose infection occurred in England in February 2000, yielded AFLP pattern A (Table 1).

Other patterns were generated by 19 of the 20 additional isolates (designated as types $\mathrm{E}-\mathrm{J}$ and $\mathrm{L}-$ W), which were not related to drug users (Table 2). The remaining $C$. novyi isolate from a leg abrasion of a patient in the UK who died during 2000 yielded AFLP pattern A (Table 2).

Those cultures with the closest similarities to AFLP type $\mathrm{A}$ in the multi-dimensional scaling diagram (and therefore of most similar patterns) were the Wellcome Bacterial Collection cultures CN 930 and CN 902, plus NCTC 6738 and NCTC 13029.

\section{C. perfringens cultures}

Forty $C$. perfringens cultures from 13 drug users, the residue from one syringe and two samples of heroin 

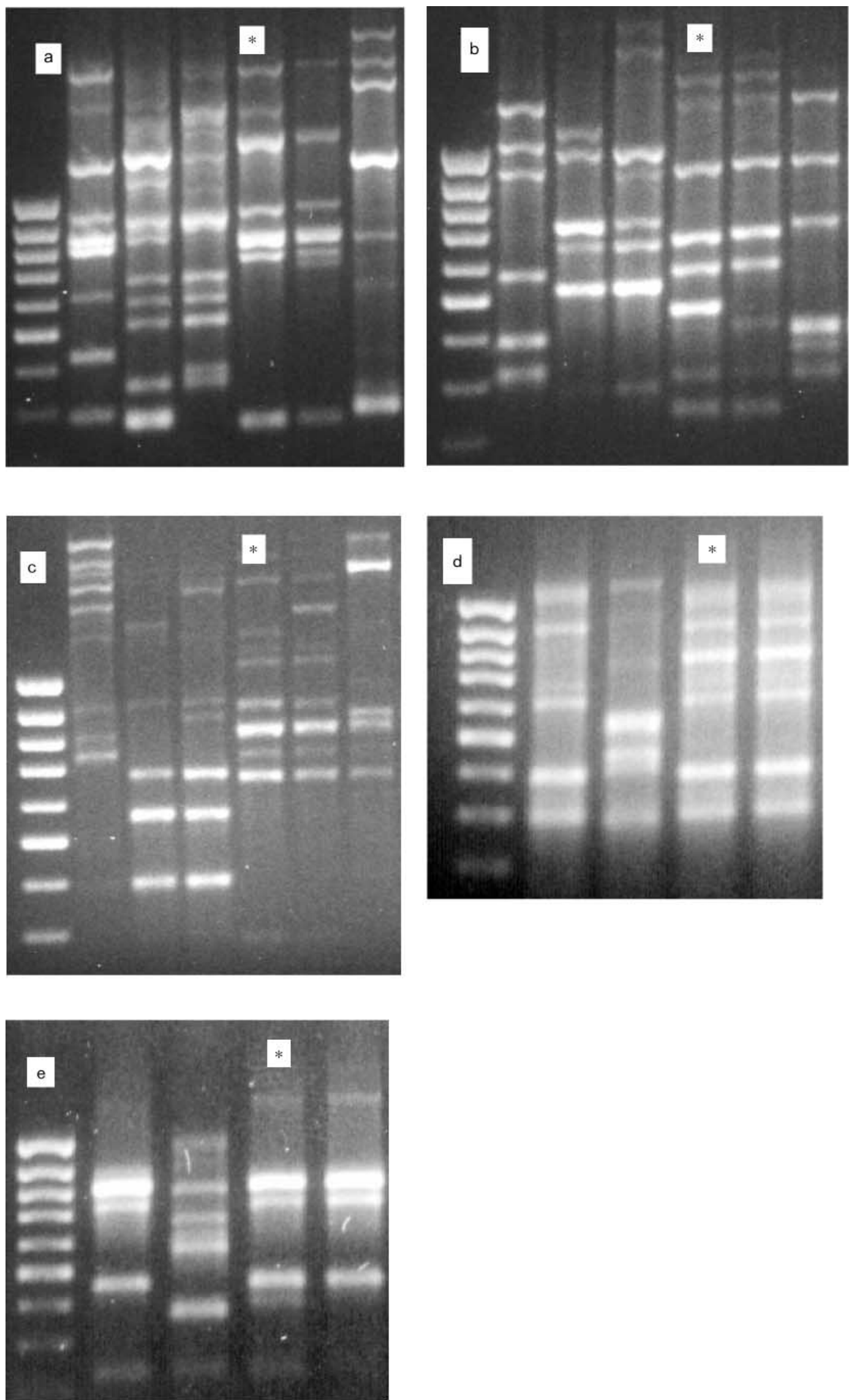

Fig. 1. Electrophoresis gels of representative C. novyi AFLP banding patterns. Primers used were: a, EC-A; b, EC-C; c, EC-T; d, HI-C; e, HI-G. * Isolates with AFLP type A. Molecular weight markers are shown on the left side of each gel. 


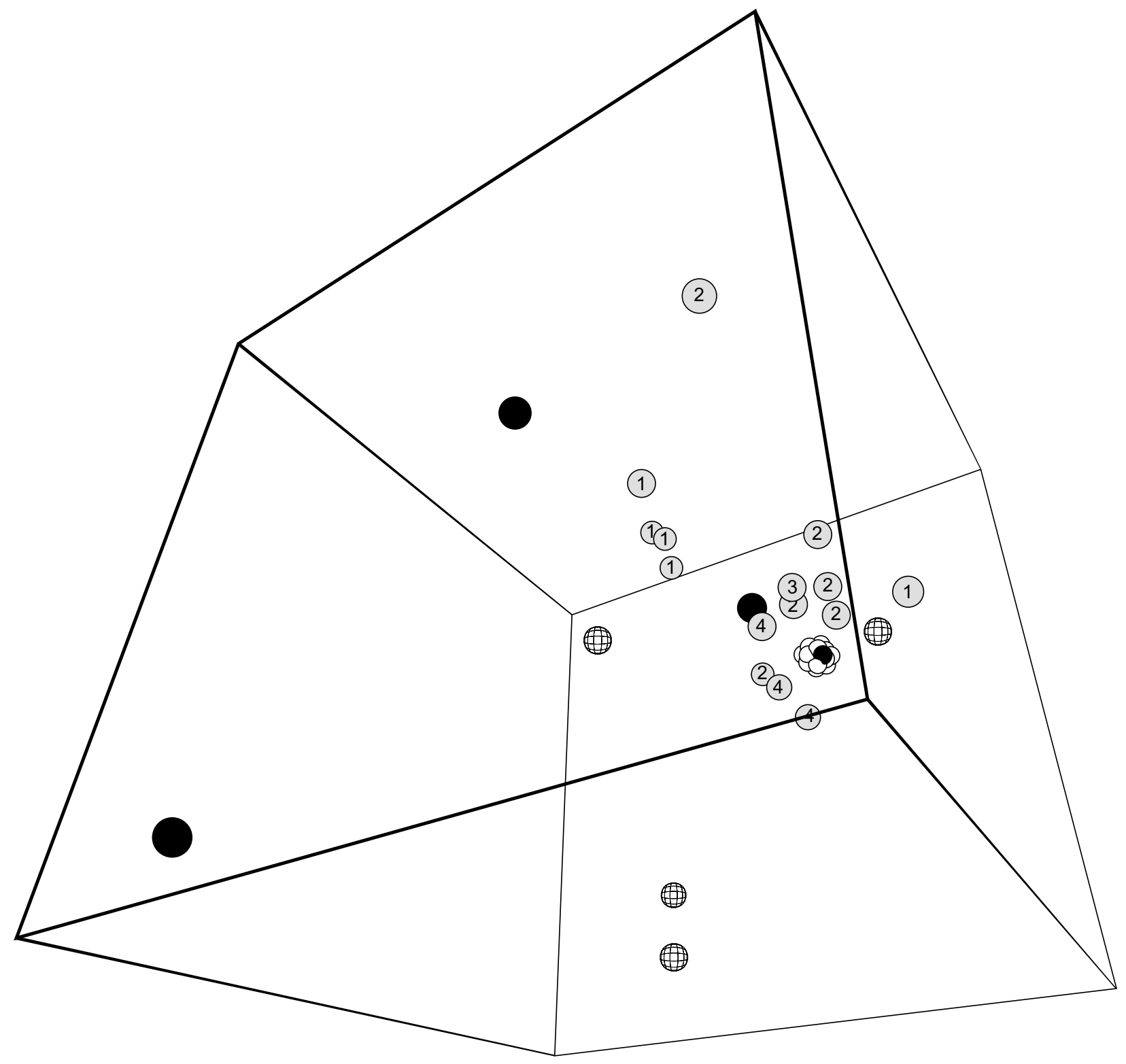

Fig. 2. Multi-dimensional scaling diagram of C. novyi AFLP based on a combined set of five similarity matrices obtained from primers HI-C, HI-G, EC-A, EC-C and EC-T. Sources of isolates: $\bigcirc$, drug users, AFLP type A; $\bigcirc$, drug users, other AFLP types; Q, other human infections; (1), animals; (2), historical isolates from human injections (Wellcome collection); (3), food; (4), NCTC cultures. One of the historical isolates is hidden from view.

were analysed. Among the cultures from the 13 drug users, 6 were collected during life, 4 at necropsy, and the remainder could not be classified. One of the heroin samples was obtained in Scotland as part of the investigations of the April-August outbreak [15]. The isolates from the syringe contents and the second heroin sample were associated with deaths in drug users but were not known to be associated with this outbreak.

A fragment of the $\alpha$-toxin was amplified from DNA isolated from all 40 cultures (further confirming their identity as $C$. perfringens), and all were negative for the enterotoxin gene fragment except for one of the two isolates from patient 27.
Nineteen (48\%) of the 40 isolates were serotypable and nine different serotypes were detected among these (Table 3). Multiple isolates were available from eight sources: three of the sources (cases 15, 18 and 27 plus the syringe residue) all yielded non-serotypable cultures, and the remaining four sources (cases 1, 17 and 33 plus the heroin from England) yielded typable as well as non-typable isolates.

AFLP analysis was performed and the banding patterns were assigned to 16 groups based on $>70 \%$ UPGMA/ Dice similarity. The same AFLP types were detected among isolates from five of the eight sources where multiple isolates were tested, i.e., those from patients 15,17 and 33, as well as the syringe contents and the 
heroin isolates in England. Of the remaining three patients from whom multiple isolates were tested (cases 8, 18 and 27), different AFLP types were obtained. The two isolates from patient 27 were already recognised as genetically different because a fragment of the enterotoxin gene was identified from one of them. Isolates from patient 8 were collected at necropsy, but the isolates from patients 18 and 27 were collected during life. Among the 16 C. perfringens isolates from patient 8 , four different types were recognised, i.e., AFLP type h serotype 18,29 (one isolate), AFLP type b serotypes TW8 and non-typable (10 isolates), AFLP type c serotypes 30,33 and non-typable (four isolates) and AFLP type c serotype TW8 (one isolate). There was almost complete agreement in the groups of cultures identified by serotyping and AFLP analysis, e.g., eight of the nine isolates from patient 8 identified as serotype TW8 were classified as AFLP type $b$ and both the heroin serotype 25 isolates were of AFLP type $\mathrm{f}$.

Among the 13 drug users, six were classified as definite, four as probable and one as possible cases; there was no information on the remaining two cases. Among the $16 C$. perfringens AFLP types, 14 were unique to their individual sources; this included the AFLP type $\mathrm{d}$ which was recognised only in the $C$. perfringens isolate from heroin examined in Scotland during the investigation of drug-related infections. The remaining two AFLP types were common to two groups: AFLP type a was found in isolates from patients 15 and 27 and the syringe residues, and AFLP type $\mathrm{c}$ in patients 8,26 and 27. Despite the similar clinical presentation in these patients, overall results from both AFLP and serotyping indicated that these $C$. perfringens isolates were diverse. This was in marked contrast to the data with respect to the lack of diversity among the $C$. novyi cultures from drug users.

\section{B. cereus cultures}

Six isolates of $B$. cereus were tested, four from drug users and two from heroin (Table 4). One of the heroin samples was collected from patient 2 [18]. The second isolate from heroin was obtained from a sample collected in England during 2000 but was not known to be associated with infections in England or Scotland. All cultures except one (patient 3) were non-typable by serotyping, and four distinct AFLP patterns were identified (Table 4). Identical AFLP patterns were obtained from the isolates from the blood and heroin of patient 2 [18], and this pattern differed by only one band from that obtained from patient 4 (Table 4). Previous analysis has indicated that a single band difference is within the limit of reproducibility of this system for $B$. cereus [27] and hence the pattern from patient 2 is designated $\mathrm{II}^{-1}$ to indicate this difference. No other common AFLP patterns were recognised among the six isolates.

\section{Discussion}

C. novyi, C. perfringens and B. cereus are widespread in soil and in the environment [39]. Furthermore, $C$. perfringens is common as part of the intestinal microflora of animals and man, indeed this bacterium is probably the most widely occurring bacterial pathogen in the environment [39]. Hence, reliable typing techniques for these three bacterial species are essential for the tracking of strains in the environment and recognising their occurrence in isolates from infected patients. The purpose of this study was to apply molecular typing techniques and, where available, conventional phenotypic characterisation tests to $C$. novyi, $C$. perfringens and $B$. cereus isolates obtained during 2000 from cases of infection among illegal drug injectors. There have been no previous reports of the characterisation of endospore-forming bacteria associated with infections in injecting drug users; hence, baseline date for comparison with this study are not available.

Although serotyping schemes are available for $B$. cereus and $C$. perfringens, these systems were both developed originally for analysis of isolates from incidents of food poisoning [31-34, 37, 38]. In the present study, both $B$. cereus and $C$. perfringens generated a high proportion of non-serotypable results, hence these schemes are not ideally suited for analysis of cultures from the sources described here. No typing systems have been described previously for $C$. novyi type A.

A previous report described the application of AFLP analysis to cultures of $B$. cereus and $C$. perfringens $[26,27]$ and it was encouraging that the method was equally suitable for AFLP analysis of a previously untested species, i.e., C. novyi.

The characterisation of two B. cereus isolates from the blood and heroin of an individual patient in Scotland during 2000 by serotyping and AFLP analysis together with sequence information on three separate genes has been reported previously [18], and these two isolates were found to be indistinguishable by AFLP. In the present study, these isolates were found to differ from those recovered from two other cases of infection among injecting drug users and another sample of heroin, strengthening the causal association between the heroin and the blood culture isolates described previously [18]. Previous analysis by AFLP of $B$. cereus from incidents of food poisoning suggested that considerable variation in this bacterium can be detected by this technique [27]. Hence, it is intriguing that $B$. cereus isolates of indistinguishable AFLP profiles were obtained from two cases of infection among drug users, both of which occurred in the same geographical region of Scotland in June 2000. A common causal link by exposure to the same contaminated source is possible for these two patients. 
Previous analysis of $C$. perfringens from cases of food poisoning by AFLP suggested that genetic diversity in this species could be detected by this technique [26]. The present report supports the use of this technique to analyse $C$. perfringens from other sources. Among the 40 C. perfringens isolates tested, variation in type was detected by both serotyping and AFLP analysis. However, serotyping has the considerable drawback that non-typable $C$. perfringens cultures occur and this can be associated with 'roughness' resulting from loss of the capsular antigen [33]. Both serotypable and nonserotypable cultures can be analysed by AFLP and, in this study where multiple cultures were obtained from a common source, the same strains can be identified by AFLP. However, there was almost complete agreement in the groups of cultures identified by serotyping and AFLP techniques, albeit that an incomplete analysis was obtained by serotyping because of the high proportion of non-serotypable isolates.

A diverse range of $C$. perfringens types were recognised from the drug users examined here and there was very limited commonality between the patients' isolates despite having a similar clinical presentation. Because of the widespread distribution of this species, several strains of $C$. perfringens may be present simultaneously in both environmental samples and an individual patient's faeces [39]. Contamination of post-mortem tissue by $C$. perfringens from the patient's own gut contents is well recognised [40]. Some of the cultures were obtained at necropsy, so this finding may reflect that of the patient's own flora and not that in the infected lesion. However, as isolates were also obtained from samples taken during life, these may have derived from a contaminated source associated with drug injection as well as from the patient's own microflora. The AFLP data, and to a lesser extent the results from serotyping, did not suggest that either a single strain of $C$. perfringens was involved with the clinical presentation or that there was contamination of a source common to the drug users investigated here.

In contrast to the AFLP results for C. perfringens, AFLP analysis of $C$. novyi indicated that all except one of the isolates from injecting drug users during the outbreak period comprised a homogeneous group. Because other typing systems are not available for $C$. novyi, confirmation of this observation by an independent method was not possible. However, it is probable that these results reflect a truly homogeneous group because identical AFLP results were obtained on replicate testing and with two different restriction endonucleases together with five different PCR primers. Furthermore, partial confirmation of this grouping was obtained by $16 \mathrm{~S}$ rDNA sequencing in that all of the AFLP type A were $100 \%$ identical to EMBL X68188 (NCTC 538). However, among 19 cultures of other AFLP types, 5 showed differences from the EMBL sequence (Brazier et al., unpublished data).
Of the C. novyi isolates cultured from cases defined as definite, all yielded AFLP profiles of type A, and this finding raises the possibility that the April-August outbreak may have started earlier than previously suspected, because one of these cases occurred in England in February 2000. The classification of all cases due to AFLP type A as probable or definite may be complicated by early medical and surgical intervention and increased awareness of the clinical presentation during the course of the outbreak, resulting in the curtailment of subsequent toxin-mediated clinical deterioration. However, with the exception of a single clinical isolate from a non-drug user in 2000, all of the patients infected with $C$. novyi AFLP type A presented with severe illness (classified as definite or probable). In contrast, the infections in the four other drug users whose $C$. novyi isolates were of four different and distinct AFLP types were classified as possible or probable, suggesting that $C$. novyi of AFLP type A may have biological differences when compared with other strains described here. Information was available for two of the four $C$. novyi cultures which were identified as having the closest similarities to AFLP type A, and both of these (CN 930 and $\mathrm{CN}$ 902) were from cases of severe infection.

The considerable interest of both the clinical and lay communities may have led to ascertainment bias in the examination of soft tissue infection (particularly for the isolation of strict anaerobic bacteria), especially among injecting drug users during the outbreak period. The increased awareness may have led to more detailed identification of Clostridium spp. than would otherwise have been performed. However, despite the increased awareness of soft tissue infections in drug users due to Clostridium spp., the consistent finding among definite cases of a single $C$. novyi AFLP type A adds to clinical and epidemiological observations about this outbreak. Investigation of the single non-drug injector who died and was infected with C. novyi AFLP type A did not reveal additional risk factors, although this individual was a keen gardener and had frequent contact with horses and domestic animals [41]. It is intriguing that this patient yielded the only other source of this AFLP type, and was resident within the UK at the same time as infections in drug users occurred. This observation highlights the need for intensive microbiological investigation (including strict anaerobic culture) for the investigation of patients with unexplained morbidity and mortality.

The majority of cases in this outbreak may have been linked by a common source contaminated by $C$. novyi AFLP type A. However, C. novyi was not isolated from any sources associated with drug injection, including heroin seized during 2000 [15, 42]. Alternatively, contamination of multiple $C$. novyi strains may have occurred, followed by clonal selection of a single $C$. novyi AFLP type which either had an adaptive advantage in spore germination, survival or growth 
following the drug preparation and injection procedure, or produced a more severe clinical presentation. The molecular epidemiological analysis reported here cannot distinguish between either a common source outbreak or clonal selection of $C$. novyi with specific virulence or survival properties; both possibilities are consistent with the data presented here.

The investigation of this whole outbreak was a collaborative effort by the Greater Glasgow Health Board, Scottish Centre for Infection and Environmental Health, Eastern Regional Health Authority (Ireland), National Disease Surveillance Centre (Ireland), Public Health Laboratory Service (UK), Centers for Disease Control and Prevention (USA); Centre for Applied Microbiology and Research, Wiltshire, and numerous public health agencies and clinical institutions in Scotland, Ireland, Norway, England and Wales. The members of the International Outbreak Investigation Team included: J. Lingappa, K. Murray, S. Reagan, S. Zaki, W-J. Shieh, J. Guarner, H. Holmes, D. Whaley, R. Weyant, R. Meyer, M. Bowen, T. Popovic, L. Mayer, S. Maslanka, J. Tappero, B. Perkins, S. Ahmed, L. Gruer, C. McGuigan, G. Penrice, K. Roberts, J. Hood, P. Redding, G. Edwards, M. Black, J. McFarlane, D. Cromie, H. Howie, A. Leonard, D. Goldberg, A. Taylor, S. Hutchinson, K. Roy, S. Wadd, R. Andraghetti, J. Barry, G. Sayers, M. Cronin, T. O'Connell, M. Ward, P. O'Sullivan, B. O'Herlihy, E. Keenan, J. O'Connor, L. Mullen, B. Sweeney, D. O'Flanagan, D. Igoe, C. Bergin, S. O'Briain, C. Keane, E. Mulvihill, P. Plunkett, G. McMahon, T. Boyle, S. Clarke, E. Leen, J. Connolly, M. Cassidy, T. Djuretic, N. Gill, V. Hope, J. Jones, G. Nichols, A. Weild, R. George, P. Borriello, J. Brazier, B.I. Duerden, J. Salmon, N. Lightfoot, A. Roberts, J. McLauchlin, M. Brett. The contributions of other PHLS staff are gratefully acknowledged including C.F.L. Amar, O. Mapamugo and V. Mithani of the Food Safety Microbiology Laboratory, V. Hall of the PHLS Anaerobe Reference Unit, and other staff in the Central Public Health Laboratory and Communicable Disease Surveillance Centre. We also thank the following clinicians and microbiologists for providing information and permission to report on patients in their care: Dr E. Mulvihill, St James's Hospital Dublin; Dr E. Smythe, Beaumont Hospital, Dublin; Dr T. Maggs, Torbay Hospital, Torquay; Dr J.M. Stockley, Worcester Royal Infirmary; Dr S. Dancer, A.H.M. Nassar and D. McNair, Vale of Leven District Hospital, Alexandria; Dr T.M.S. Reid, Microbiology Department Aberdeen Royal Infirmary; Dr E. Kaczmaski, Manchester PHL; Dr E. Davies, North Bristol NHS Trust; Dr P. Redding, Victoria Infirmary, Glasgow; Dr M. Noone, J.B. Spillane and T. Tabaqchali, North Tees General Hospital, Stockton on Tees; Dr C. Lafong and J. Malone, Victoria Hospital, Fife; Dr J.S. Cheesbrough, Preston PHL; Dr S. Hill, Poole PHL; Dr A. Stacey, Reading PHL; Dr A. Leanord, Monklands District General Hospital, Airdrie; Dr J. Paul, Brighton PHL; Dr S. Samavedam, Western Infirmary Glasgow; Dr G. Edwards, Stobhill Hospital, Glasgow; Dr E.A. Hoiby, National Institute of Public Health, Oslo.

\section{References}

1. Brettle RP. Infection and injection drug use. $J$ Infect 1992; 25: 121-131.

2. Horsburgh CR, Anderson JR, Boyko EJ. Increased incidence of infections in intravenous drug users. Infect Control Hosp Epidemiol 1989; 10: 211-215.

3. Peat M, Budd J, Burns SM, Robertson R. Audit of blood borne virus infections in injecting drug users in general practice. Commun Dis Public Health 2000; 3: 244-246.

4. Martin C, Schaller MD, Lepori M, Liaudet L. Cranial nerve palsies and decending paralysis in a drug abuser resulting from wound botulism. Intensive Care Med 1999; 25: 765-769.

5. Werner SB, Passaro D, McGee J, Schechter R, Vugia DJ. Wound botulism in California, 1951-1998: recent epidemic in heroin injectors. Clin Infect Dis 2000; 31: 1018-1024.

6. Jensenius M, Lovstad RZ, Dhaenens G, Rorvik LM. A heroin user with a wobbly head. Lancet 2000; 356: 1160.

7. Talan DA, Moran GJ. Tetanus among injecting drug users: California 1997. Ann Emerg Med 1998; 32: 385-386.

8. Ringertz SH, Hoiby EA, Jensenius $M$ et al. Injectional anthrax in a heroin skin-popper. Lancet 2000; 354: 1574-1575.

9. Bloomfield SF, Arthur M. Mechanisms of inactivation and resistance of spores to chemical biocides. Soc Appl Bacteriol Symp Suppl 1994; 23: 91S-104S

10. Christie B. Gangrene bug "killed 35 heroin users". BMJ 2000; 320: 1690.

11. Anon. Serious unexplained illness among drug injectors. Commun Dis Rep CDR Wkly 2000; 24: 195.

12. Anon. Clostridium novyi is likely cause of 'serious unexplained illness' as cases continue to be reported. Commun Dis Rep CDR Wkly 2000; 24: 213, 216.

13. Anon. Update: Clostridium novyi and unexplained illness among injecting drug users: Scotland, Ireland and England April-June 2000. MMWR Morb Mortal Wkly Rep 2000; 49: $543-544$.

14. Ryan JM, Paul J, Curtis S, Patel NK. Clostridium novyi infection: a fatal association with injecting drug users. Emerg Med $J$ 2001; 18: 138-139.

15. McGuigan CC, Penrice GM, Black $\mathrm{M}$ et al. Outbreak of infection with Clostridium novyi and other sporulating bacteria in Scottish injecting drug users. $J$ Med Microbiol 2002; 51: 971-977.

16. Hood J, Horne G, Sweeney G, Baird K. Third case of wound botulism in an injecting drug user. SCIEH Weekly Report 2000; 34: 200 .

17. Mulleague L, Bonner SM, Samuel A et al. Wound botulism in drug addicts in the United Kingdom. Anaesthesia 2001; 56: $120-123$.

18. Dancer SJ, McNair D, Finn P, Kolstot AB. Bacillus cereus cellulitis from contaminated heroin. $J$ Med Microbiol 2002; 51: $278-281$.

19. Athwal BS, Gale AN, Brett MM, Youl BD. Wound botulism in UK. Lancet 2000; 356: 2001-2002.

20. Khan M, Chay S, Bonner S, Brett M. Wound botulism in injecting drug users: second case in England. Eurosurveillance Weekly 2000; 22 June.

21. Zabeau M, Vos P. Selective restriction fragment amplification: a general method for DNA fingerprinting. Munich, European Patent Office. No. EP 0534 858, 1993.

22. Vos P, Hogers R, Bleeker M et al. AFLP: a new technique for DNA fingerprinting. Nucleic Acids Res 1995; 23: 4407-4414.

23. Janssen P, Coopman R, Huys G et al. Evaluation of the DNA fingerprinting method AFLP as a new tool in bacterial taxonomy. Microbiology 1996; 142: 1881-1893.

24. Gibson JR, Slater E, Xerry J, Tompkins DS, Owen RJ. Use of an amplified-fragment length polymorphism technique to fingerprint and differentiate isolates of Helicobacter pylori. $J$ Clin Microbiol 1998; 36: 2580-2585.

25. Fry NK, Bangsborg JM, Bernander S et al. Assessment of intercentre reproducibility and epidemiological concordance of Legionella pneumophila serogroup 1 genotyping by amplified fragment length polymorphism analysis. Eur J Clin Microbiol Infect Dis 2000; 19: 773-780.

26. McLauchlin J, Ripabelli G, Brett MM, Threlfall EJ. Amplified fragment length polymorphism (AFLP) analysis of Clostridium perfringens for epidemiological typing. Int J Food Microbiol 2000; 56: $21-28$.

27. Ripabelli G, McLauchlin J, Mithani V, Threlfall EJ. Epidemiological typing of Bacillus cereus by amplified fragment length polymorphism. Lett Appl Microbiol 2000; 30: 358-363.

28. Maagaard A, Hermansen N, Heger B et al. Serious systemic illness among injecting drug users in Europe: new case in Oslo. Euroserveillance Weekly 2000; 14 September.

29. Holdeman LV, Moore WEC, Cato EP (eds). Anaerobe laboratory manual, 4th edn. Blacksburg, VA, Virginia Polytechnic Institute and State University. 1977.

30. Hutson RA, Thompson DE, Collins MD. Genetic interrelationships of saccharolytic Clostridium botulinum types B, E and F and related clostridia as revealed by small-subunit rRNA gene sequences. FEMS Microbiol Lett 1993; 108: 103-110.

31. Berry PR, Gilbert RJ. Clostridium perfringens type A food poisoning. In: Duerden BI, Draser BS (eds) Anaerobes in human disease. London, Arnold. 1991: 362-371.

32. Stringer MF, Turnbull PCB, Gilbert RJ. Application of serological typing to the investigation of outbreaks of Clostridium perfringens food poisoning, 1970-1978. J Hyg 1980; 84: 443-456.

33. Stringer MF, Watson GN, Gilbert RJ. Clostridium perfringens type A: serological typing and methods for the detection of enterotoxin. In: Corry JEL, Roberts D, Skinner FA (eds) Isolation and identification methods for food poisoning 
organisms. Society for Applied Bacteriology Technical Series, no. 17. London, Academic Press. 1982: 111-135.

34. Stringer MF, Watson GN, Gilbert RJ et al. Faecal carriage of Clostridium perfringens. J Hyg 1985; 95: 277-288.

35. Fach P, Popoff MR. Detection of enterotoxigenic Clostridium perfringens in food and faecal samples with a duplex PCR and the slide latex agglutination test. Appl Environ Microbiol 1997; 63: $4232-4236$

36. Parry JM, Turnball PCB, Gibson JR. A colour atlas of Bacillus species. London, Wolf Medical Publications. 1983.

37. Taylor AJ, Gilbert RJ. Bacillus cereus food poisoning: a provisional serotyping scheme. J Med Microbiol 1975; 8: 543-550.

38. Gilbert RJ, Parry JM. Serotypes of Bacillus cereus from outbreaks of food poisoning and from routine foods. $J \mathrm{Hyg}$ 1977; 78: $69-74$.

39. Hatheway CL, Johnson EA. Clostridium: the spore-bearing anaerobes. In: Balows A, Duerden BI (eds) Topley and Wilson's microbiology and microbial infections, 9th edn, vol 2. Systematic bacteriology. London, Arnold. 1998: 731-782.

40. Koneman EW, Davis MA. Postmortem bacteriology. III. Clinical significance of microorganisms recovered at autopsy. Am J Clin Pathol 1974; 61: 28-40.

41. McGuigan C, Roworth M. Clostridium novyi type A infection: a sporadic fatal case. Scand J Infect Dis 2002; 34: 209-211.

42. McLaughlin J, Mithani V, Bolton FJ et al. An investigation into the microflora of heroin. J Med Microbiol 2002; 51: 1001-1008. 